\title{
A SAXS-WAXS study of the endothermic transitions in amorphous or supercooled liquid Itraconazole
}

\author{
C.J. Benmore ${ }^{1,2}$, Q.Mou ${ }^{2,5}$, K.J. Benmore ${ }^{3}$, D.S. Robinson ${ }^{1}$, J. Neuefeind ${ }^{6}$, \\ J. llavsky ${ }^{1}$, S.R. Byrn ${ }^{4}$ and J.L. Yarger ${ }^{2,5}$
}

1) X-ray Science Division, Advanced Photon Source, Argonne National Laboratory, $9700 \mathrm{~S}$. Cass Avenue, Illinois 60439

2) Department of Physics, Arizona State University, Tempe, AZ 85287

3) Department of Materials Science and Engineering, University of Sheffield, Sheffield, S1 3JD

4) Department of Industrial and Physical Pharmacy, Purdue University, West Lafayette, Indiana

5) Magnetic Resonance Research Center, Arizona State University, Tempe, AZ 85287

6) Spallation Neutron Source, Oak Ridge National Laboratory, Tennessee 37830.

\section{ABSTRACT.}

Small and wide angle high energy $\mathrm{x}$-ray scattering experiments were performed upon cooling itraconazole from the melt to investigate the structural origin of the two transitions at $\sim 74^{\circ} \mathrm{C}$ and $\sim 90^{\circ} \mathrm{C}$ observed in DSC measurements. Slight changes to the main WAXS peak at $\mathrm{Q}=1.33 \pm 0.01 \AA^{-1}$ were observed at $90^{\circ} \mathrm{C}$ and are found to be inter-molecular in nature, suggesting an isotropic liquid to isotropic transition. This finding was supported by complementary wide angle neutron scattering measurements. For temperatures at and below $\sim 74^{\circ} \mathrm{C}$ two strong rings appear in the 2D-SAXS pattern at $\mathrm{Q}=0.24 \pm 0.01 \AA^{-1}$ and $0.43 \pm 0.01 \AA^{-1}$. The SAXS spectra were further deconvoluted into sharp and broad components. A narrowing of the broad component is associated with only minor changes in the packing arrangements of the itraconazole molecules below $\sim 90^{\circ} \mathrm{C}$, while the appearance of the sharp component below $\sim 74^{\circ} \mathrm{C}$ is attributed to the formation of a polydomain lamellar phase.

\section{INTRODUCTION.}

Itraconazole is an effective antifungal drug, but it exhibits a relatively poor dissolution rate and low bioavailability after oral administration $[1,2]$. Itraconazole can exist in different disordered forms (mesophases) that are distinct from its' crystal structure. Characterizing the structures of the different forms of pharmaceuticals is an important first step in optimizing a molecules' solubility and predicting stability. Itraconazole has a rod-like molecular structure [3] and modulated differential scanning calorimetry measurements have revealed two endothermic transitions at $74^{\circ} \mathrm{C}$ and $90^{\circ} \mathrm{C}$ (above the glass transition temperature of $59^{\circ} \mathrm{C}$ ) [1]. Upon cooling from the melt a color change occurs from transparent to white at $90^{\circ} \mathrm{C}$ [2]. Six et al. [1] have suggested an amorphous chiral nematic mesophase forms upon cooling from the liquid state, in which layers of molecules pack asymmetrically to form a twisted structure, 
followed by limited rotational mobility within the molecule. Alternatively, based on FTIR measurements it has been suggested that homeotropic alignment of the rigid rod-like molecular cores occurs [2], and that a nematic phase exists below $90^{\circ} \mathrm{C}$ (whereby the rods are roughly aligned but no planes are formed) and a smetic $\mathrm{A}$ phase arranges below $74^{\circ} \mathrm{C}$ in which the parallel rods are layered along the normal direction. Previous X-ray diffraction studies have demonstrated a strong temperature dependence of the diffuse scattering signal of the principal peak [2], and that variable degrees of ordering occur at low-Q values on samples quenched from different temperatures [1]. To test these predictions here we have performed in-situ high energy $\mathrm{x}$-ray diffraction measurements on itraconazole encompassing a wide Q-range, as a function of temperature. The diffuse scattering data has been analyzed using a combination of small and wide angle $x$-ray scattering (SAXS-WAXS) to identify the structural rearrangements associated with the two endothermic transitions.

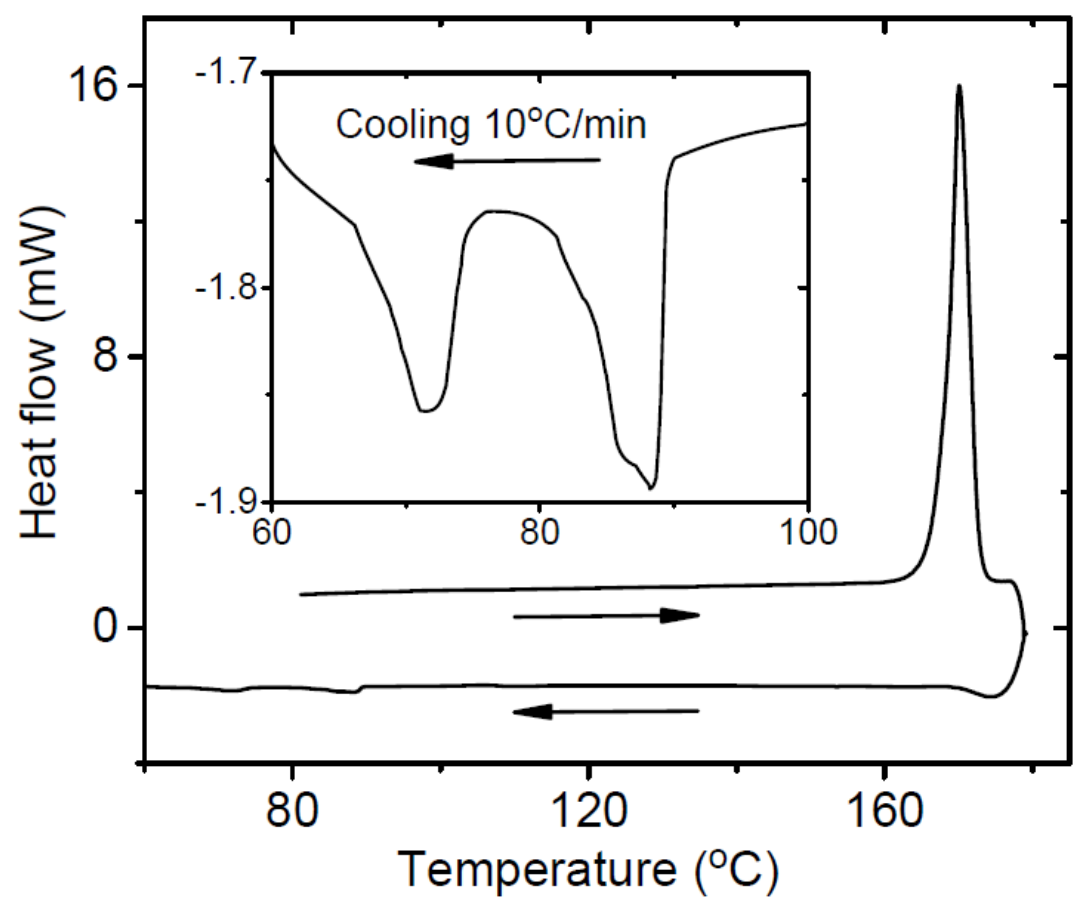

Figure 1. Differential Scanning Calorimetry measurement for itraconazole upon heating to the melt and cooling. Insert: Expanded region of the trace upon cooling from the melt shows transitions at $\sim 90^{\circ} \mathrm{C}$ and $\sim 74^{\circ} \mathrm{C}$ (see text).

\section{EXPERIMENTAL}

Differential scanning calorimetry measurements were performed using a DSC 2910 (Du Pont Instruments and associated software) calorimeter on itraconazole powder (Sigma, Lot $\# 097 \mathrm{~K} 1156 \mathrm{~V}$, purity $>99 \%$ as determined by thin layer chromatography and a racemic mixture chirality) at a cooling rate of $10^{\circ} \mathrm{C} / \mathrm{min}$. Samples were sealed into aluminum pans and placed in the DSC, purged with helium gas. Calibration was conducted using the onset temperature of an indium standard, which has a melting point of $156.6^{\circ} \mathrm{C}$. The itraconazole samples were melted at $180^{\circ} \mathrm{C}$ for 5 minutes and cooled to room temperature in a controlled manner using liquid 
nitrogen to give transition temperatures in the supercooled liquid of $\mathrm{T}_{\text {onset }}=73.7+/-0.1^{0} \mathrm{C}$ $\left(T_{\text {peak }}=74.3+/-0.1^{\circ} \mathrm{C}\right)$ and $T_{\text {onset }}=89.0+/-0.1^{\circ} \mathrm{C}\left(T_{\text {peak }}=89.4+/-0.1^{\circ} \mathrm{C}\right)$. All errors quoted are standard uncertainties. The transition temperature values compare well to those previously reported in the literature of $74^{\circ} \mathrm{C}$ and $90^{\circ} \mathrm{C}[1]$ and $73^{\circ} \mathrm{C}$ and $90^{\circ} \mathrm{C}$ [2].

High energy x-ray (WAXS) experiments were performed on beamline 6-ID-D at the Advanced Photon source using an incident energy of $100.00 \mathrm{keV}(\lambda=0.1236 \AA$ ). The sample detector distance was $554 \mathrm{~mm}$ and the Q-scale was calibrated with a $\mathrm{CeO}_{2}$ powder standard. The same batch of itraconazole powder was loaded into a $2 \mathrm{~mm}$ diameter thin walled $(100 \mu \mathrm{m})$ capillary and heated in a furnace to $180^{\circ} \mathrm{C}$, just above the melting point $\left(\mathrm{T}_{\mathrm{m}}=166^{\circ} \mathrm{C}\right)$ for 3 minutes before cooling to $109^{\circ} \mathrm{C}$. Thirty minute scans were then performed every $2^{\circ} \mathrm{C}$ upon cooling from 109 to $41^{\circ} \mathrm{C}\left( \pm 0.1^{\circ} \mathrm{C}\right)$ and the scattered $\mathrm{x}$-ray intensity measured using a Perkin Elmer XRD1621 2D area detector. Standard x-ray source (polarization, Q-calibration), sample (attenuation, multiple and Compton scattering, form factor) and detector (dark current, oblique incidence etc.) dependent corrections have been applied, to extract the x-ray structure factor $S_{X}(Q)$ [4]. Similarly, wide angle neutron scattering (WANS) experiments were performed on itraconazole at $227^{\circ} \mathrm{C}, 100^{\circ} \mathrm{C}, 80^{\circ} \mathrm{C}$ and $40^{\circ} \mathrm{C}\left( \pm 1^{\circ} \mathrm{C}\right)$ using the Nanoscale Ordered Materials Diffractometer (NOMAD) at the Spallation Neutron Source [5]. Crystalline Itraconazole was loaded into a $3 \mathrm{~mm}$ diameter quartz capillary at room temperature and mounted on a translation stage that allows the fine positioning into the neutron beam. The temperature was controlled with an Ar gas stream produced by an Cobra cryosystem from Oxford Instruments. Data collection was made at the four elevated temperatures for an integrated accelerator proton charge of $3.610^{12} \mathrm{pC}$, for slightly longer than 1 hour at the relevant accelerator power. Additional measurements of the empty quartz capillary, a vanadium sample, a diamond sample and the empty diffractometer were made for background subtraction, calibration and normalization.

To further probe the longer range correlations in itraconazole SAXS measurements were performed using a PIXIRAD detector on beamline 6-ID-D with an incident energy of 70keV. The sample to detector distance was $1725 \mathrm{~mm}$ and the absolute Q-scale was calibrated with Silver Behenate. The same sample heating and cooling procedure was followed as for the WAXS measurements, but with only two 5 minute scans at each temperature. A noticeable difference in instrumental resolution was observed in the overlap region between the SAXS and WAXS measurements (see figure 2). Attenuation and background subtraction measurements performed to extract the $x$-ray intensity from the sample $I_{x}(Q)$. 


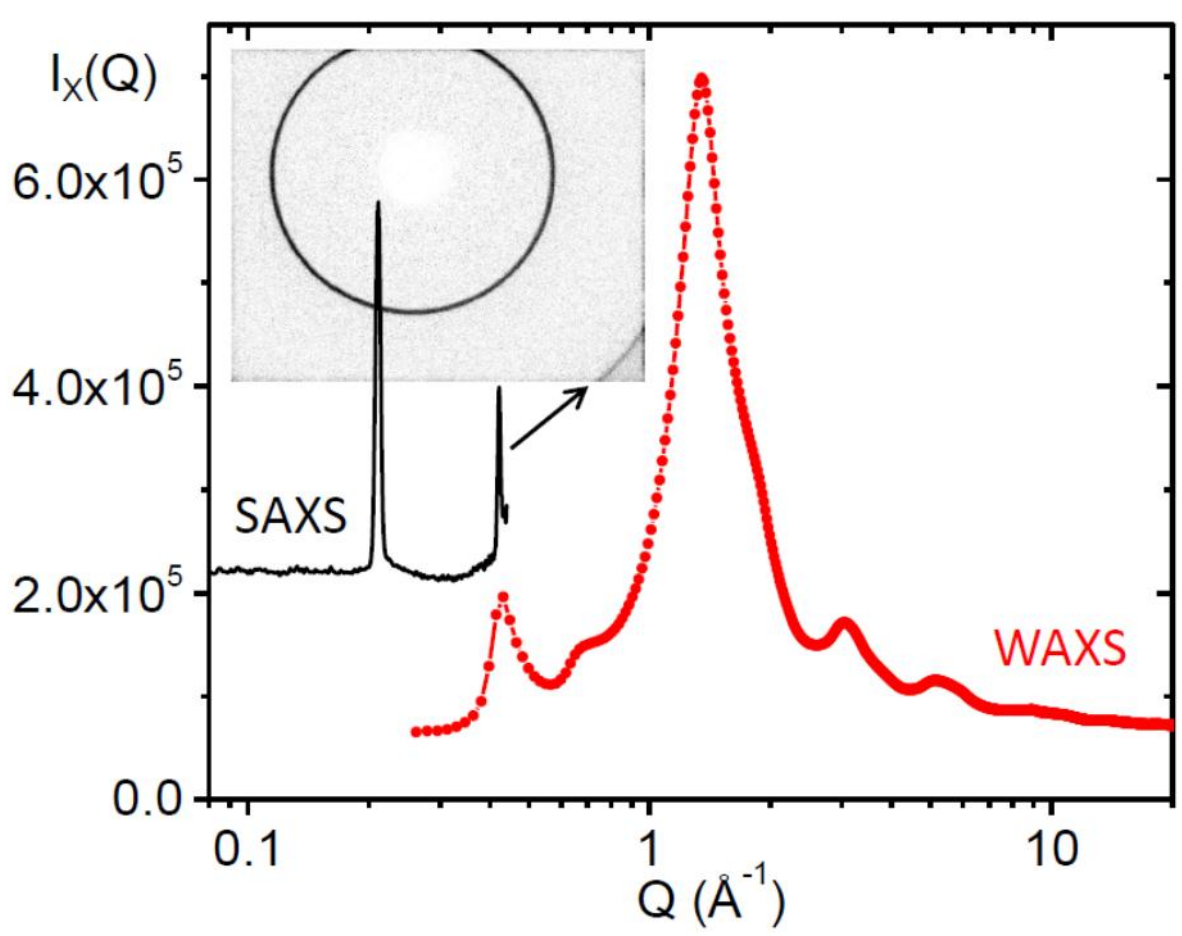

Figure 2. The SAXS and WAXS data collected on amorphous Itraconazole at 300K. The SAXS data is displaced for clarity and the insert illustrates the 2D pattern collected on the PIXIRAD detector corrected for the $\sqrt{3} / 2$ triangular arrangement of hexagonal pixels. The peak at $Q=0.43 \pm 0.01 \AA^{-1}$ is only measured in the bottom right corner of the 2D-SAXS image.

\section{RESULTS AND DATA ANALYSIS}

For disordered molecular materials it is useful to separate the WAXS total structure factor into the contributions arising from intra-molecular and inter-molecular scattering [4];

$$
S_{X}(Q)=S_{\text {intra }}(Q)+S_{\text {inter }}(Q)
$$

The inter-molecular function usually decays rapidly at high-Q values except for any short-range interactions. A multi-parameter fit is required to separate out the intra-molecular scattering contribution from the measured structure factor. This has previously been achieved by iterative methods for simple molecules containing only a few atoms i.e. $<10$. For itraconazole, which contains 87 atoms, initial distance parameters were obtained by fits to the crystal structure [6] and the Debye-Waller thermal factors $\sigma_{\alpha \beta}$ are estimated within an empirical algorithm. Details of our algorithm for fitting the intra-molecular x-ray scattering are given in references [7] and [8]. The crystal structure of itraconazole is triclinic and there are 4 molecules in each unit cell volume of $3447.3 \AA^{-3}$ [6]. Each molecule contains seven rings in which the triazole and benzene rings are essentially planar, but there two conformations of the methoxy group which are either orientated perpendicular or almost co-planar to the phenyl ring. There are no intermolecular contacts significantly shorter than the sum of the van der Waals radii [9]. 
The fits to our preliminary $\mathrm{x}$-ray data on supercooled liquid and amorphous Itraconazole indicate the molecular conformation of the rigid rod-like structure is the same and that the main inter-molecular structural changes occur around and below the principal peak in the WAXS pattern. The rod-like intra-molecular structure of the itraconazole molecule was fitted to the x-ray structure factors over the Q-range 4.5 to $20.6 \AA^{-1}$ using our XISF software routine [7]. The residual errors were $0.43 \%, 0.37 \%, 0.31 \%$ at temperatures of $61^{\circ} \mathrm{C}, 81^{\circ} \mathrm{C}$ and $100^{\circ} \mathrm{C}$ respectively.
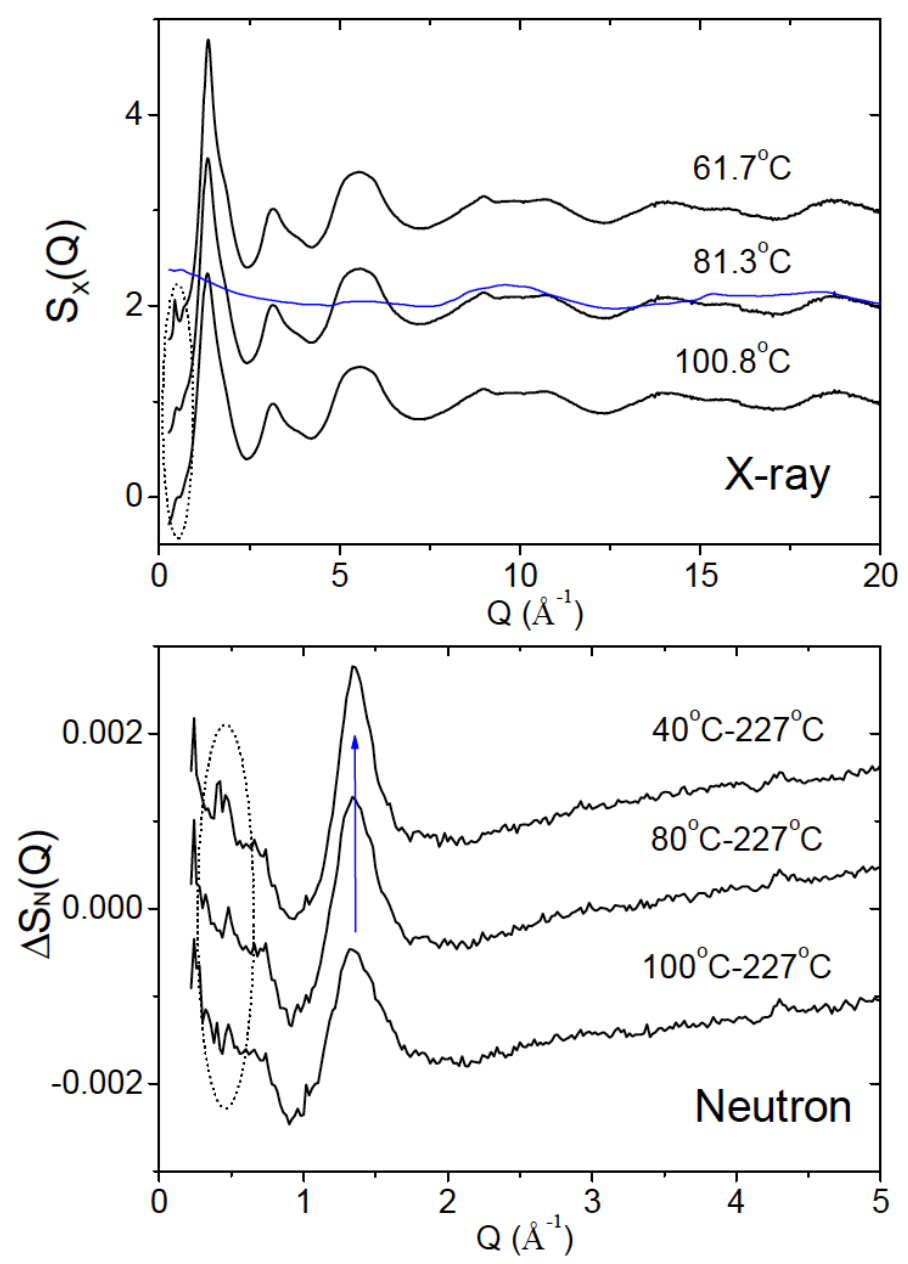

Figure 3. Top. Measured WAXS structure factor of itraconazole at selected temperatures. A representative intra-molecular fit to the structure factor $S_{\text {inter }}(Q)$ is shown for $T=81.3 \pm 0.01^{\circ} \mathrm{C}$ (blue curve). As the temperature increases, the XISF program yields a lower residual error indicating a better fit to the data. Bottom. Differences between wide angle neutron (WANS) structure factors taken at different temperatures on itraconazole cooled from the melt show small changes in the low-Q region (circled) in agreement with the WAXS data above. 


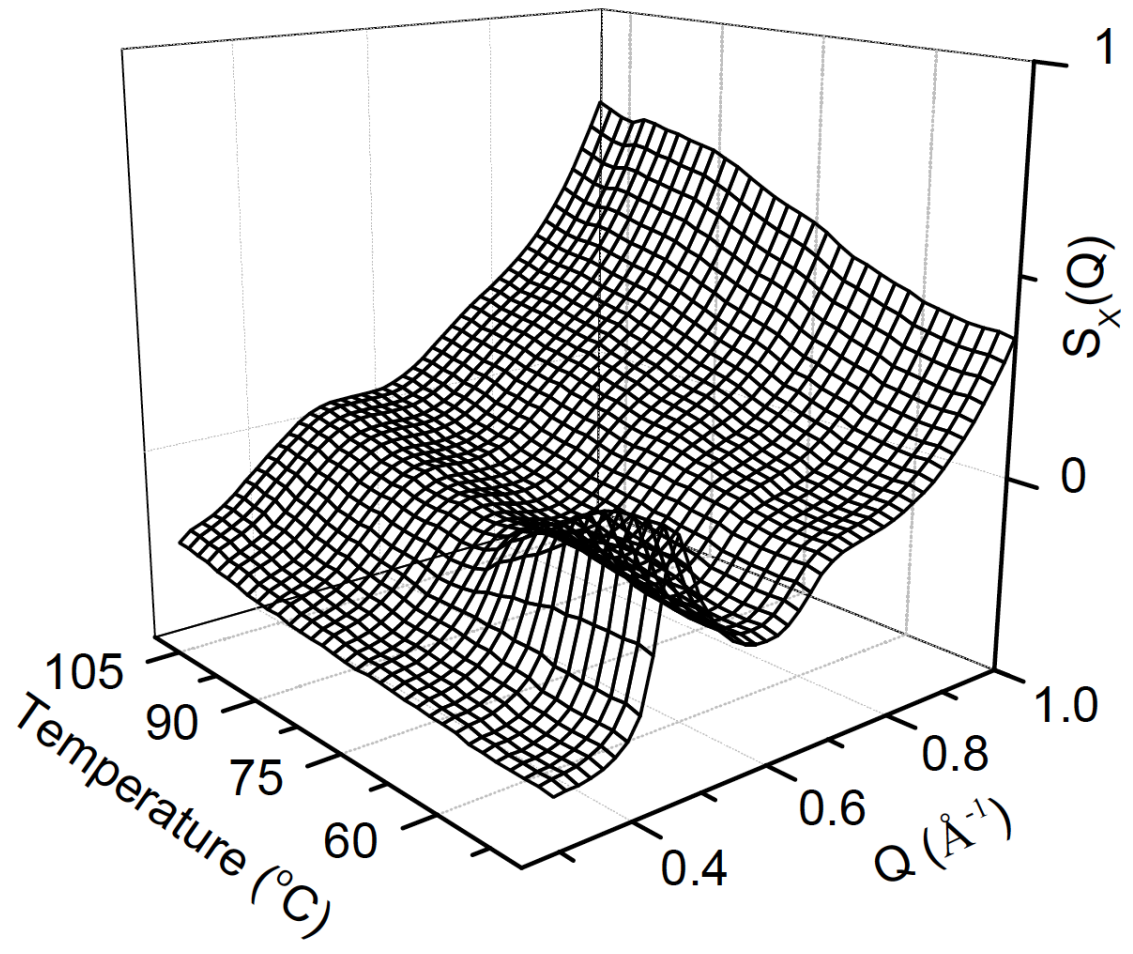

Figure 4. Changes in the wide angle $x$-ray structure factor at the lowest $Q$-values upon cooling from the melt. This plot highlights the small features shown in the top figure that appear before the principal peak. The peak at $Q=0.43 \pm 0.01 \AA^{-1}$ begins at $\sim 74^{\circ} \mathrm{C}$, whereas the smaller feature at $\mathrm{Q}=0.7 \pm 0.03 \AA^{-1}$ varies with temperature.

Differences between adjacent 2D WAXS diffraction patterns upon cooling from the melt indicate that significant changes occur within principal peak at $Q=1.33 \pm 0.01 \AA^{-1}$, which becomes asymmetric around the two transitions temperatures of $74^{\circ} \mathrm{C}$ and $90^{\circ} \mathrm{C}$. The molecular shape at high-Q is invariant through the two transitions. Similar behavior, including a sharpening of the peak at $Q=1.33 \AA^{-1}$, also occurs in the neutron scattering data (figure 3 bottom).

Figure 3 shows the WAXS and WANS structure factors for both the liquid and different amorphous forms itraconazole and that the main changes occur in the low-Q region which are expanded in figure 4 . The transition at $T \sim 74^{\circ} \mathrm{C}$ is associated with the appearance of the symmetric ring at $Q=0.43 \pm 0.01 \AA^{-1}$ in the $2 D$ WAXS diffraction pattern. A weaker feature at $Q=0.7 \pm 0.03 \AA^{-1}$ also sharpens and moves slightly towards lower- $Q$ values upon cooling. However, the smaller pixel size and longer sample to detector distance in the SAXS experiment resulted in a much higher resolution measurement of the $Q=0.43 \AA^{-1}$ peak. The SAXS data also reveal the appearance of an intense low-Q peak at $0.24 \pm 0.01 \AA^{-1}$ at and below the transition temperature of $\sim 74^{\circ} \mathrm{C}$, just beyond the normal Q-range of WAXS measurements. An analysis of the $Q=0.24 \AA^{-1}$ feature shows that the SAXS signal can be de-convolved into two Gaussian components for temperatures $\leq 74^{\circ} \mathrm{C}$ (see figure 5). For $\mathrm{T}>74^{\circ} \mathrm{C}$ only the broad peak exists. The position of the sharp and broad peaks as a function of temperature is shown in figure 4 . Below 
$\sim 60^{\circ} \mathrm{C}$ the sharp peak tends to a value of $\mathrm{Q}=0.210 \pm 0.001 \AA^{-1}$ and the broad peak tends to a value of $Q=0.218 \pm 0.001 \AA^{-1}$.
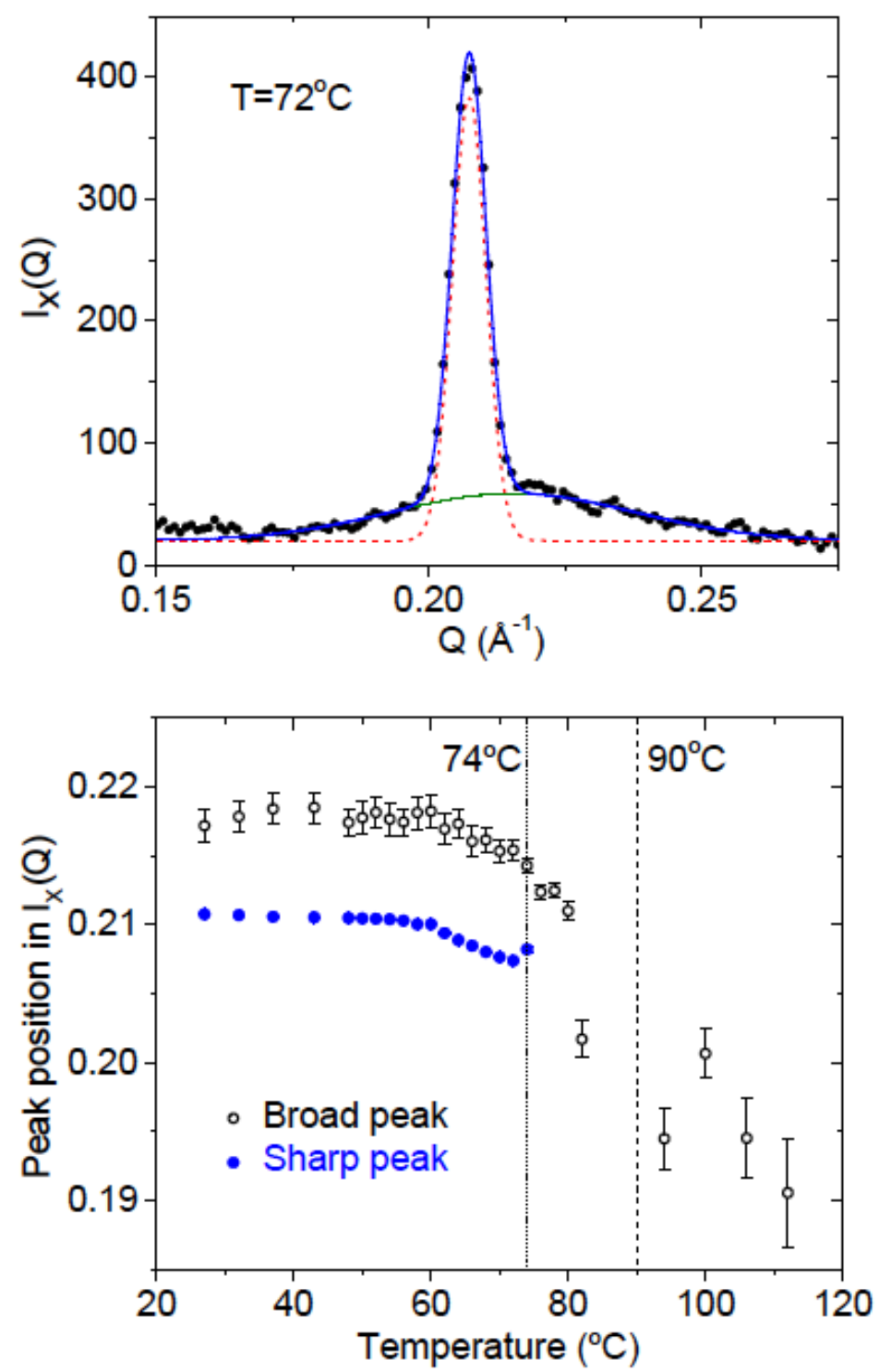

Figure 5. Top. Measured SAXS intensity for itraconazole at $72 \pm 0.1^{\circ} \mathrm{C}$ (filled circles) fitted with two Gaussian curves showing a sharp (red dashed line) and broad (green dotted line) component to the data. The total fit is shown by the solid blue line. Bottom. The variation in peak position of the two Gaussian curve fits illustrated above; broad peak (open circles) and sharp peak (filled blue circles). The sharp component only appears at temperatures $\leq 74{ }^{\circ} \mathrm{C}$. 

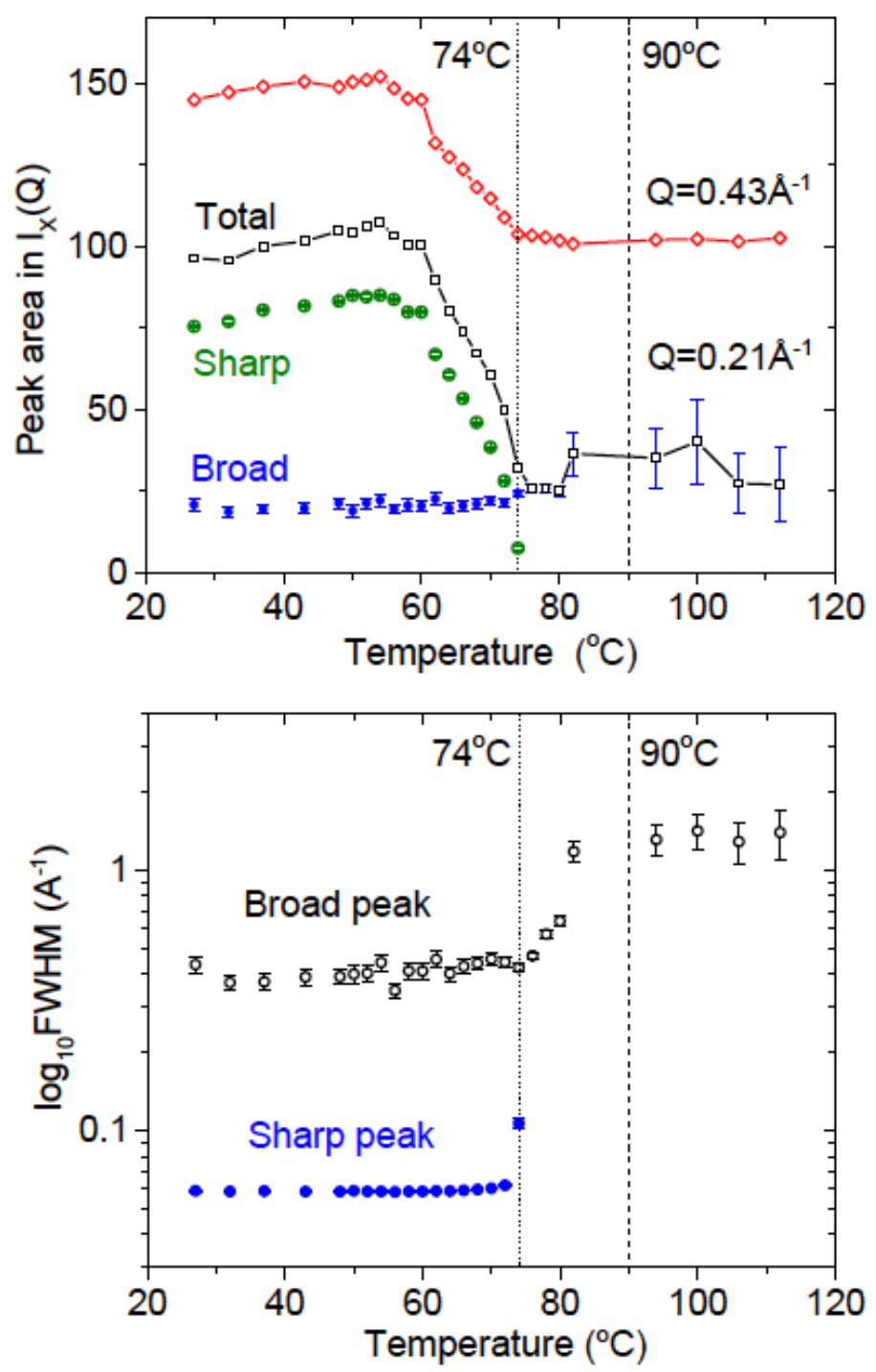

Figure 6. Top. Areas of the SAXS peaks at $Q=0.24 \pm 0.01 \AA^{-1}$ (diamonds and red line) and $Q=0.43 \pm 0.01 \AA^{-1}$ (squares and black line) as a function of temperature. The areas of the two Gaussian components for the $Q=0.24 \AA^{-1}$ peak are also shown; sharp peak (green circles at temperatures $\leq 74^{\circ} \mathrm{C}$ ) and broad peak (blue circles). Bottom. The full width half maximum (FWHM) of the sharp peak (closed blue circles) and broad peak (open circles) components as a function of temperature.

The growth in total area of the peaks at $Q=0.24 \AA^{-1}$ and $Q=0.43 \AA^{-1}$ below $74^{\circ} \mathrm{C}$ is given in figure 6 . Both peak areas increase linearly with decreasing temperature between $74^{\circ} \mathrm{C}$ and $60^{\circ} \mathrm{C}$ and plateau to room temperature. A breakdown of the two Gaussian components for the $\mathrm{Q}=0.24 \AA^{-1}$ peak show that the area of the broad component is essentially invariant with temperature while the sharp peak area dominates the temperature dependent changes. The peak full width half maximum (FWHM) of the sharp component below $\sim 74^{\circ} \mathrm{C}$ is invariant at 
$\Delta \mathrm{Q}=0.06 \AA^{-1}$, whereas the broad peak width starts to decrease below $\sim 80^{\circ} \mathrm{C}$ and plateaus below $74^{\circ} \mathrm{C}$ at $\triangle \mathrm{Q}=0.4 \AA^{-1}$.

\section{DISCUSSION}

The main changes observed at the $90^{\circ} \mathrm{C}$ transition correspond to subtle variations in the principal peak of the WAXS and WANS data. In addition, the broad peak component seen in the SAXS data shows a narrowing below $90^{\circ} \mathrm{C}$. These structural changes all point towards the transition from the melt to isotropic amorphous phase being associated with a subtle packing change of the itraconazole rods. In a typical aligned liquid crystal nematic phase the 2D diffraction pattern is concentrated into symmetric lobes. However the $90^{\circ} \mathrm{C}$ scattering data and low-Q rings in the $2 \mathrm{D}$ SAXS $x$-ray pattern appearing at $74^{\circ} \mathrm{C}$ upon cooling from the melt, indicate a polydomain liquid crystal structure with varying directors, $n$. The room temperature peak positions at $Q=0.24 \AA^{-1}, 0.43 \AA^{-1}$ and $0.7 \AA^{-1}$ correspond to ordering with periodicities of $2 \pi / Q=\sim 26.2 \AA, \sim 14.6 \AA$ and $\sim 9 \AA$ respectively.

We note that the approximate effective length of an itraconazole molecule is $\sim 16.4 \AA$ (reflecting $x$-rays insensitivity to hydrogen atoms) and the common shortest intermolecular distance between molecules is $\sim 3.7 \AA$ in the crystal. Since smallest angle peak observed (at $Q=0.24 \AA^{-1}$ ) is the only feature that has a periodicity larger than the effective length of an itraconazole molecule it is most likely associated with short range positional order between nearest neighbour molecules along the direction parallel to $n$ [10]. The approximate 2:1 distance ratio between the first two peaks indicates the presence of a lamellar phase [10], in which small bunches of rod-like molecules are aligned end-on, along the $\mathrm{n}$ direction (see figure 7). The extent of the layering requires measurements at smaller scattering angles to be performed. The larger angle $\left(Q=0.43 \AA^{-1}\right.$ and $\left.0.7 \AA^{-1}\right)$ peaks in the liquid crystal x-ray diffraction patterns are consequently assumed to be associated with short range positional order between nearest neighbor molecules along the direction normal to the director, $\mathrm{n}$ [10].

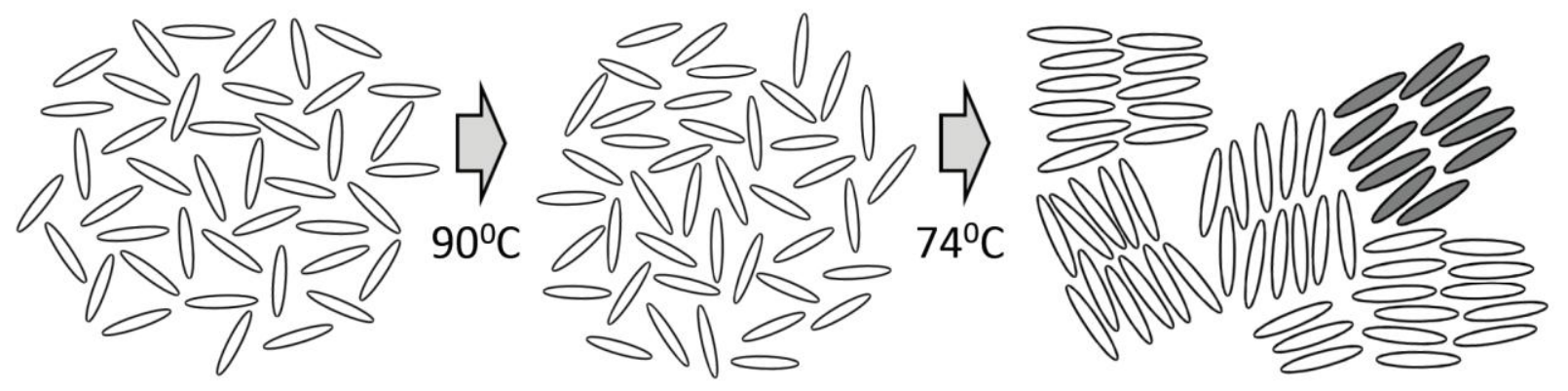

Figure 7. Schematic diagram of the structural ordering that occurs upon cooling itraconazole based on the SAXS-WAXS data.

\section{CONCLUSIONS}


Two amorphous or supercooled liquid forms of itraconazole have been studied using SAXS-WAXS experiments upon cooling from the melt. Changes in the principal WAXS and WANS peaks and a narrowing of a broad component in the SAXS data are observed upon passing through the first transition at $90^{\circ} \mathrm{C}$, indicating an liquid to isotropic transition associated with only a subtle changes in the packing of the itraconazole molecules. However two strong rings appear in the SAXS signal at the second transition of $74^{\circ} \mathrm{C}$ indicating the transformation to a highly ordered polydomain lamellar phase.

\section{Acknowledgements.}

This work was supported by the Office of Basic Energy Sciences, U.S. Department of Energy, at the Advanced Photon Source, Argonne National Laboratory under contract number DE-AC02-06CH1135 and at the Spallation neutron Source, Oak Ridge National Laboratory under contract DE-AC05-00OR22725 with UT Battelle.

\section{References.}

[1] K. Six, G. Verreck, J. Peeters, K. Binnemans, H. Berghmans. P. Augustijns, R. Kinget, G. Van den Mooter. Investigation of thermal properties of glassy itraconazole: identification of a monotropic mesophase. Thermochimica Acta 376, 175 (2001).

[2] F. Atassi, R.J. Behme, P.J. Patel. Mesomorphous versus traces of crystallinity: the itraconazole example.Thermochimica Acta 574, 133 (2013).

[3] M. Tarnacka, K. Adrjanowicz, E. Kaminski, K. Grzybowska, K. Kolodziejczyk, P. Wlodarczyk, L. Hawelek, G. Garbacz, A Kocot, M. Paluch. Molecular dynamics of itraconazole at ambient and high pressure. Phys. Chem. Chem. Phys. 15, 20742 (2013).

[4] C.J. Benmore, J.K.R. Weber, A.N. Tailor, B. Cherry, J.L. Yarger, Q. Mou, W. Weber, J. Neuefeind, S.R. Byrn, Structural characterization and aging of glassy pharmaceuticals made using acoustic levitation. J. Pharm. Sci., 102, 1290 (2013).

[5] J. Neuefeind, M. Feygenson, J. Carruth, R. Hoffmann, K. Chipley, The Nanoscale Ordered Materials Diffractometer NOMAD at the Spallation Neutron Source SNS. Nuc. Instr. Meth. B 287, 68 (2012).

[6] O. M. Peeters, N. M. Blaton, and C. J. De Ranter. cis-2-sec-Butyl-4-\{4-[4-(4-\{[2-(2,4dichlorophenyl)-2-(1H-1,2,4-triazol-1-ylmethyl)-1,3-dioxolan-4-yl]methoxy\}phenyl)-1piperazinyl]phenyl\}-2,4-dihydro-3H-1,2,4-triazol-3-one (Itraconazole). Acta Crystallogr., C52, 2225 (1996).

[7] Q. Mou, C.J. Benmore, J.L. Yarger, XISF - Separation of intra- and inter- molecular interactions from total X-ray scattering data. J. Appl. Cryst. 48, 950 (2015).

[8] J.K.R. Weber, C.J. Benmore, A.N. Tailor, S.K. Tumber, J. Neuefeind, B. Cherry, J.L. Yarger, Q. Mou, W. Weber and S.R. Byrn. Neutron-X-ray and Calorimetric Study of Glassy Probucol Synthesized Using Containerless Techniques. Chemical Physics, 424, 89 (2013).

[9] A. A. Al-Badr and H.I. El-Subbagh. Chapter 5 itraconazole: comprehensive profile. Profiles of Drug Substances, Excipients, and Related Methodology 34 (2009) 193. Elsevier Inc. ISSN 00995428. 
[10] P. Davidson, D. Petermann, A.M. Levelut. The measurement of the nematic order parameter by x-ray scattering reconsidered. J. Phys. II France 5, 113 (1995) 